\title{
O uso do canabidiol (CBD) no tratamento da doença de Parkinson e suas comorbidades
}

\author{
The use of cannabidiol (CBD) in the treatment of Parkinson's disease \\ and its comorbidities
}

\author{
Rafael G. dos Santos ${ }^{1}$, Jaime E. C. Hallak ${ }^{1}$, José Alexandre S. Crippa ${ }^{1}$
}

dos Santos RG, Hallak JEC, Crippa JAS. O uso do canabidiol (CBD) no tratamento da doença de Parkinson e suas comorbidades / The
use of cannabidiol (CBD) in the treatment of Parkinson's disease and its comorbidities. Rev Med (São Paulo). 2019 jan.-fev.;98(1):46-51.

RESUMO: Fundamento: A doença de Parkinson (DP) é um transtorno neurodegenerativo crônico caracterizado por sintomas motores (bradicinesia, tremores, rigidez) e não motores (transtornos psicóticos, do humor e do sono). Os tratamentos farmacológicos disponíveis não são eficazes para uma parcela significativa dos pacientes. Pesquisas recentes sugerem que o fitocanabinóide canabidiol (CBD) poderia ser eficaz no tratamento de alguns sintomas da DP. Objetivos: Revisar os estudos préclínicos e clínicos dos efeitos do CBD nos sintomas da DP. Métodos: Revisão narrativa dos principais estudos básicos e clínicos sobre os efeitos do CBD nos sintomas da DP. Devido ao número pequeno de estudos clínicos, pesquisas com outros canabinóides também foram incluídas. Resultados: No total, 15 artigos foram incluídos na revisão: 5 estudos pré-clínicos envolvendo a administração de CBD e 10 estudos com pacientes envolvendo a administração de diferentes canabinóides (maconha, nabilona, CBD). A maioria dos estudos básicos mostraram um efeito positivo do CBD em comportamentos ou alterações bioquímicas relacionadas à DP. Estudos observacionais e clínicos com uso de maconha fumada, extrato oral de maconha, nabilona (canabinóide sintético) e CBD sugerem que estes canabinóides podem reduzir sintomas motores (bradicinesia, tremores, rigidez) e não motores (transtornos psicóticos, do humor e do sono, qualidade de vida) da DP. Além disso, são substâncias bem toleradas e com poucos efeitos adversos significativos. Conclusão: Embora o CBD tenha demonstrado resultados favoráveis tanto em estudos pré-clínicos como em estudos clínicos, estas evidências ainda não são suficientes para indicar o uso deste canabinóide em pacientes com distúrbios do DP. Novos estudos controlados devem ser realizados com diferentes dosagens de CBD para replicar estes dados.

Descritores: Doença de Parkinson; Canabinóides; Canabidiol.

\begin{abstract}
Background: Parkinson's disease (PD) is a chronic neurodegenerative disorder characterized by motor (bradykinesia, tremors, stiffness) and non-motor (psychotic, mood and sleep disorders) symptoms. The available pharmacological treatments are not effective for a significant portion of the patients. Recent research suggests that the phytocannabinoid cannabidiol (CBD) could be effective in treating some PD symptoms. Objectives: To review the preclinical and clinical studies of the effects of CBD on the symptoms of PD. Methods: Narrative review of the main basic and clinical studies on the effects of CBD on the symptoms of PD. Due to the small number of clinical studies, research with other cannabinoids was also included. Results: A total of 15 articles were included in the review: 5 preclinical studies involving the administration of CBD and 10 studies with patients involving the administration of different cannabinoids (cannabis, nabilone, CBD). Most of the basic studies reported a positive effect of CBD on PD-related behavior or biochemical changes. Observational and clinical studies using smoked cannabis, oral cannabis extract, nabilone (synthetic cannabinoid) and CBD suggest that these cannabinoids can reduce motor (bradykinesia, tremors, stiffness) and non-motor (psychotic, mood and sleep disorders, quality of life) symptoms of PD. Moreover, they are well tolerated substances with few significant adverse effects. Conclusion: Although CBD has shown favorable results in both preclinical and clinical studies, this evidence is not yet sufficient to indicate the use of this cannabinoid in patients with PD. New controlled studies should be performed with different dosages of CBD to replicate this data.
\end{abstract}

Keywords: Parkinson disease; Cannabinoids; Cannabidiol.

Este trabalho foi realizado no Departamento de Neurociências e Ciências do Comportamento, Faculdade de Medicina de Ribeirão Preto, Universidade de São Paulo.

1. Department of Neurosciences and Behavior, Ribeirão Preto Medical School, University of São Paulo, Ribeirão Preto, São Paulo, Brazil. National Institute of Science and Technology - Translational Medicine, Ribeirão Preto, São Paulo, Brazil. ORCID: dos Santos RG - https://orcid.org/00000003-2388-4745; Hallak JEC - https://orcid.org/0000-0002-8784-0189; Crippa JAS - https://orcid.org/0000-0001-9520-6746. Email: banisteria@ gmail.com; jhallak@fmrp.usp.br; jcrippa@fmrp.usp.br.

Endereço para correspondência: Prof. Rafael G. dos Santos, PhD. Departamento de Neurociências e Ciências do Comportamento, Faculdade de Medicina de Ribeirão Preto, Universidade de São Paulo, Hospital das Clínicas, Terceiro Andar. Av. Bandeirantes, 3900. Ribeirão Preto, São Paulo, Brazil. E-mail: banisteria@gmail.com. 


\section{INTRODUÇÃO}

doença de Parkinson (DP) é um transtorno neurodegenerativo crônico com incidência de $1-2 \%$ na população mundial acima de 65 anos, e com prevalência estimada de $3,3 \%$ no Brasil, sendo caracterizado por sintomas motores típicos como bradicinesia (lentidão dos movimentos), tremores e rigidez, além de sintomas neuropsiquiátricos como transtornos psicóticos, do humor e do sono ${ }^{1-8}$. A DP é causada pela degeneração de neurônios que produzem o neurotransmissor dopamina em uma região cerebral chamada gânglios da base, especificamente na região denominada substância negra, o que resulta na redução da quantidade de dopamina em outra área cerebral, o corpo estriado ${ }^{1-10}$.

O tratamento farmacológico para pacientes com DP envolve basicamente a administração de precursores da dopamina como a levodopa (L-DOPA), mas em uma proporção significativa dos pacientes estas medicações diminuem seu efeito terapêutico com o passar do tempo, produzindo reações adversas graves como a discinesia tardia (movimentos involuntários) $)^{1-10}$.

Receptores canabinóides (receptor canabinóide 1 e 2 ou $\mathrm{CB}_{1}$ e $\mathrm{CB}_{2}$ ) e compostos endocanabinóides ( $N$-aracdonoil etanolamina ou anandamida, e 2-aracdonoil glicerol ou 2-AG) são encontrados em grandes concentrações em áreas cerebrais envolvidas no processamento e execução de movimentos corporais, como, por exemplo, os gânglios da base $\mathrm{e}^{11-19}$, e estudos em animais e humanos demonstram que o sistema endocanabinóide passa por alterações neuroquímicas durante a evolução da DP, incluindo redução na quantidade de receptores $\mathrm{CB}_{1}$ nas fases iniciais da doença e aumento da expressão destes receptores (e também do receptor canabinóide $2, \mathrm{CB}_{2}$ ) e de endocanabinóides nas fases intermediárias e mais avançadas da $\mathrm{DP}^{11-19}$, o que sugere um possível uso terapêutico dos fitocanabinóides (delta-9-tetraidrocanabinol, THC; canabidiol, CBD; etc.) no tratamento desta doença ${ }^{19-28}$. Estudos pré-clínicos também sugerem que, dependendo da fase da doença e das diferentes subáreas dos gânglios da base envolvidas, os canabinóides podem modular as complexas alterações neuroquímicas nos níveis dos neurotransmissores glutamato e GABA (ácido gama-aminobutírico) causadas pela redução dos níveis de dopamina na DP através da ativação ou inibição dos receptores $\mathrm{CB}_{1 / 2}{ }^{1-9,23}$.

Ademais, estudos pré-clínicos e em humanos indicam que tanto as drogas agonistas ${ }^{19,20,23,25,27-33}$ como as antagonistas ${ }^{22,24,30}$ do receptor $\mathrm{CB}_{1}$ possuem potencial uso medicinal nos sintomas motores da DP. Isso se deve provavelmente à complexidade anatômica e funcional de diferentes subáreas dos gânglios da base, da distribuição dos receptores $\mathrm{CB}_{1}$ e dos endocanabinóides nestas áreas, e das alterações na quantidade destes receptores e dos endocanabinóides em diferentes fases da DP. Estudos préclínicos também sugerem que os efeitos antiparkinsonianos dos canabinóides poderiam ser produzidos por suas propriedades antioxidantes, anti-excitotóxicas e antiinflamatórias, que parecem envolver não apenas o receptor $\mathrm{CB}_{1}$, mas também o receptor $\mathrm{CB}_{2}$ e outros mecanismos de ação que independem dos receptores canabinóides ${ }^{1-9,34-36}$.

Embora vários estudos em animais tenham demonstrado que o CBD possui uma série de efeitos terapêuticos relevantes para a DP, incluindo efeitos anti-inflamatórios, anti-excitotóxicos, antioxidantes, antipsicóticos e sedativos ${ }^{37-42}$, poucos estudos avaliaram o potencial terapêutico dos canabinóides em geral e do CBD em particular em pacientes com DP. Portanto, o objetivo desta revisão é apresentar um panorama atualizado sobre os estudos envolvendo a administração do CBD tanto em modelos animais como em ensaios clínicos.

\section{MATERIAIS E MÉTODOS}

Trata-se de uma revisão temática da literatura sobre o uso do CBD em modelos animais da DP e sobre o uso deste composto em pesquisa clínica. No caso da literatura pré-clínica, restringimos a busca a artigos experimentais in vitro e in vivo de administração de CBD em modelos específicos da DP. Já no caso da literatura clínica, incluímos estudos observacionais e experimentais apenas em pacientes (estudos com voluntários saudáveis foram excluídos), mas com outros canabinóides além do CBD, tanto sintéticos (nabilona, rimonabanto) como naturais (THC). Os artigos foram extraídos das bases eletrônicas PubMed, SciELO e LILACS por dois autores (RGS, JECL), com dúvidas sendo resolvidas por um terceiro autor (JASC). A busca incluiu artigos completos publicados até maio de 2018, excluindo cartas, editoriais, resumos e revisões. As revisões mais recentes foram incluídas na Introdução e na Discussão do artigo.

\section{RESULTADOS E DISCUSSÃO}

No total, 15 artigos foram incluídos na presente revisão: cinco estudos pré-clínicos envolvendo a administração de CBD e 10 estudos com pacientes envolvendo a administração de canabinóides (um estudo experimental com maconha, um estudo experimental com um extrato de maconha contendo THC e CBD, dois estudos experimentais com uso de nabilona, três estudos observacionais com uso de maconha, e três estudos experimentais com uso de CBD). Os resultados destes estudos são apresentados nas Tabelas 1 e 2 e são discutidos abaixo.

\section{Estudos pré-clínicos avaliando os efeitos do CBD em modelos de DP}

Um número cada vez maior de estudos pré-clínicos vem avaliando os efeitos do CBD em modelos de DP 
(Tabela 1$)^{28,32,34,43,44}$. Três estudos que utilizaram um modelo animal de parkinsonismo que consiste na depleção de dopamina no estriado de ratos após injeção da neurotoxina 6-hidroxidopamina (6-OHDA) demostraram que este efeito foi atenuado pela administração de $\mathrm{CBD}^{28,34,43}$. Por outro lado, um modelo in vitro de doença de Parkinson utilizando as neurotoxinas 1-metil-4-fenil-piridínio $\left(\mathrm{MPP}^{+}\right)$, paraquat e lactacistina em células neurais humanas relatou ausência de efeitos antiparkinsonianos do $\mathrm{CBD}^{32}$, e um estudo recente em camundongos utilizando a toxina 6-OHDA não observou efeitos do CBD quando administrado sozinho, mas quando este composto foi administrado juntamente com a capsazepina (antagonista do receptor de potencial transitório subtipo V1 ou TRPV-1), houve redução de sinais comportamentais e moleculares associados à discinesia ${ }^{44}$.

Os dados acima discutidos e resumidos na Tabela 1 sugerem que o CBD possui propriedades potencialmente benéficas para o tratamento dos sintomas da DP. Além disso, os efeitos positivos do CBD em diferentes modelos de DP sugerem que este composto poderia tratar vários sintomas utilizando diferentes mecanismos de ação. Como veremos abaixo, estes dados pré-clínicos estão sendo replicados em estudos clínicos.

Tabela 1. Efeitos do CBD em modelos pré-clínicos de doença de Parkinson

\begin{tabular}{|c|c|c|c|}
\hline Modelo & Organismo / Tecido & Resultados & Referências \\
\hline 6-OHDA & Ratos & $(+)$ & Lastres-Becker et al., $2005^{43}$ \\
\hline 6-OHDA & Ratos & $(+)$ & García-Arencibia et al., $2007^{34}$ \\
\hline 6-OHDA & Ratos & $(+)$ & García et al., $2011^{28}$ \\
\hline $\mathrm{MPP}^{+}$, paraquat, lactacistina & Células neurais humanas & $(-)$ & Carroll et al., $2012^{32}$ \\
\hline 6-OHDA/L-DOPA & Camundongos & $(-)^{2}$ & Dos Santos-Pereira et al., $2016^{44}$ \\
\hline
\end{tabular}

(+) efeito antiparkinsoniano; (-) ausência de efeito antiparkinsoniano.

L-DOPA: levodopa; 6-OHDA: 6-hidroxidopamina.

${ }^{1}$ Extrato 1:1 THC:CBD.

${ }^{2}$ Achado positivo quando o CBD foi administrado juntamente com capsazepina.

\section{Estudos clínicos avaliando os efeitos de canabinóides em pacientes com Parkinson}

Poucos estudos avaliaram os efeitos dos canabinóides em geral e do CBD em particular em pacientes com DP (Tabela 2).

Um estudo com administração de um agonista do receptor $\mathrm{CB}_{1}$ (nabilona) para sete pacientes com DP relatou uma redução significativa da discinesia ${ }^{33}$. Entretanto, outro estudo com administração de nabilona para 15 pacientes com DP e distonia não relatou efeitos terapêuticos ${ }^{45}$. Um estudo com administração de um extrato de maconha contendo THC e CBD para 19 pacientes com discinesia também não relatou efeitos benéficos ${ }^{46}$. Ademais, um estudo com administração de um antagonista do receptor $\mathrm{CB}_{1}$ (rimonabanto) para 24 pacientes com DP não observou redução dos sintomas motores e discinesia ${ }^{47}$.

Três pesquisas envolvendo entrevistas com pacientes que utilizavam maconha para tratar seus sintomas de DP descreveram que uma proporção significativa destes pacientes relatou melhoras nos sintomas gerais, bradicinesia, rigidez muscular, tremores e discinesia, além de melhoras no humor e sono ${ }^{48-50}$. Mais recentemente, um estudo aberto com 22 pacientes relatando uso medicinal de maconha descreveu reduções significativas dos sintomas motores (bradicinesia, rigidez muscular e tremores), além de diminuição da dor e melhora do sono ${ }^{51}$.
Com relação ao CBD especificamente, três estudos clínicos foram publicados (Tabela 2). Todos os estudos foram realizados por nosso grupo de pesquisa no Departamento de Neurociências e Ciências do Comportamento da Faculdade de Medicina de Ribeirão Preto (Universidade de São Paulo).

Em um estudo aberto com seis pacientes com DP e psicose, doses diárias crescentes de CBD (150-400 mg/dia) durante quatro semanas reduziram de maneira significativa os sintomas psicóticos dos pacientes, que continuaram seu tratamento farmacológico habitual durante o estudo ${ }^{52}$. Ademais, o CBD reduziu as pontuações de sintomas globais da DP e não produziu efeitos motores ou outros efeitos adversos. Em uma série de casos, quatro pacientes com DP e distúrbio comportamental do sono REM (rapid eye movement), caracterizado por sonhos intensos e vívidos, frequentemente com conteúdo agressivo, receberam CBD (75 ou $300 \mathrm{mg} /$ dia) durante seis semanas e apresentaram melhora significativa dos sintomas ${ }^{53}$. Um estudo duplocego avaliou os efeitos do CBD ( 75 ou $300 \mathrm{mg}$ /dia durante seis semanas) em pacientes com DP, relatando melhora na qualidade de vida destes pacientes, especificamente em relação às atividades diárias e ao estigma associado à doença ${ }^{54}$.

A Tabela 2 mostra os estudos de administração de CBD em pacientes com DP. 
dos Santos RG, et al. O uso do canabidiol (CBD) no tratamento da doença de Parkinson e suas comorbidades .

Tabela 2. Efeitos do CBD em pacientes com doença de Parkinson

\begin{tabular}{|c|c|c|c|}
\hline Desenho do Estudo / Dose & Pacientes & Resultados & Referências \\
\hline $\begin{array}{l}\text { Estudo aberto } \\
150-400 \mathrm{mg} / \text { dia por } 4 \text { semanas }\end{array}$ & $\begin{array}{l}6 \text { pacientes com DP e } \\
\text { psicose }\end{array}$ & $\begin{array}{l}\text { Melhora de sintomas psicóticos } \\
\text { e redução de sintomas globais do } \\
\text { Parkinson }\end{array}$ & Zuardi et al., $2009^{52}$ \\
\hline $\begin{array}{l}\text { Série de casos } \\
75 \text { ou } 300 \mathrm{mg} / \text { dia por } 6 \text { semanas }\end{array}$ & $\begin{array}{l}4 \text { pacientes com DP } \\
\text { e DCR }\end{array}$ & Melhora de sintomas do DCR & Chagas et al., $2014^{53}$ \\
\hline $\begin{array}{l}\text { Duplo-cego controlado com placebo } \\
75 \text { ou } 300 \mathrm{mg} / \text { dia por } 6 \text { semanas }\end{array}$ & 21 pacientes com DP & $\begin{array}{l}\text { Melhora na qualidade de vida global, } \\
\text { atividades diárias e estigma } \\
(300 \mathrm{mg} / \mathrm{dia})\end{array}$ & Chagas et al., $2014 \mathrm{~b}^{54}$ \\
\hline
\end{tabular}

DP, doença de Parkinson; DCR, distúrbio comportamental do sono REM (rapid eye movement).

\section{CONCLUSÕES}

Estudos pré-clínicos in vitro e in vivo utilizando diversos modelos de DP demonstram que o CBD possui propriedades antiparkinsonianas. No entanto, embora os resultados sejam promissores e o número de estudos venha crescendo, este número de estudos ainda é limitado. Além disso, todos os cinco estudos pré-clínicos utilizaram a mesma toxina (6-OHDA), limitando a extrapolação dos resultados para outros modelos. Ademais, não se sabe exatamente como o CBD produz seus efeitos antiparkinsonianos. Segundo os estudos revisados, estes efeitos seriam produzidos tanto por mecanismos de ação que envolvem os receptores $\mathrm{CB}_{1 / 2}$ como por outros mecanismos que independem destes receptores, sendo possivelmente explicados por suas propriedades antioxidantes e anti-inflamatórias, talvez por meio dos receptores TRPV-1 ou $\mathrm{VMAT}_{1 / 2}$.

No caso dos estudos clínicos, existem poucos estudos controlados com administração de canabinóides em geral e de CBD em particular. Embora o CBD tenha demonstrado resultados favoráveis tanto em estudos préclínicos como em estudos clínicos, estas evidências ainda não são suficientes para indicar o uso deste canabinóide em pacientes com distúrbios do DP. Novos estudos controlados devem ser realizados com diferentes dosagens de CBD para replicar estes dados.

No entanto, os resultados até agora encontrados sugerem que tanto a maconha in natura quanto os canabinóides isolados (sintéticos e naturais) são bem tolerados e possuem propriedades terapêuticas para o tratamento de sintomas motores (bradicinesia, rigidez muscular, tremores) e não-motores (sono, humor, ansiedade, psicose, qualidade de vida) de pacientes com DP. Portanto, novos estudos clínicos são necessários tanto com o CBD como com outros canabinóides para tentar replicar os dados pré-clínicos e clínicos até agora encontrados, além de melhorar nossa compreensão dos mecanismos de ação responsáveis por estes efeitos.

CONTRIBUIÇÃO DE CADA AUTOR: RGS - fez a pesquisa bibliográfica e escreveu a primeira versão do manuscrito. JECH e $J A S C$ fizeram a pesquisa bibliográfica e contribuíram intelectualmente para a versão final do texto.

CONFLITOS DE INTERESSE: $R G S$ é bolsista do Programa Nacional de Pós-Doutorado da CAPES (PNPD/CAPES). JECH e JASC são co-inventores (Mechoulam R, JASC, Guimarães FS, AZ, JECH, Breuer A) da patente "Fluorinated CBD compounds, compositions and uses thereof. Pub. No.: WO/2014/108899. International Application No.: PCT/IL2014/050023” Def. US no. Reg. 62193296; 29/07/2015; INPI on 19/08/2015 (BR1120150164927). A Universidade de São Paulo licenciou a patente para Phytecs Pharm (Resolução USP 15.1.130002.1.1). A Universidade de São Paulo tem um acordo com a Prati-Donaduzzi (Toledo, Brasil) para "desenvolver um produto farmacêutico contendo canabidiol sintético e comprovar sua segurança e eficácia terapêutica no tratamento da epilepsia, esquizofrenia, doença de Parkinson e transtornos de ansiedade". JECH e JASC receberam verba para viagens e são consultores médicos da BSPG-Pharm.

\section{REFERÊNCIAS}

1. Lastres-Becker I, Fernández-Ruiz J. An overview of Parkinson's disease and the cannabinoid system and possible benefits of cannabinoid-based treatments. Curr Med Chem. 2006;13(30):3705-18. doi: 10.2174/092986706779026156.

2. de Lago E, Fernández-RuizJ. Cannabinoids and neuroprotection in motor-related disorders. CNS Neurol Disord Drug Targets. 2007;6(6):377-87. doi: 10.2174/187152707783399210

3. Sagredo O, García-Arencibia M, de Lago E, Finetti S, Decio $\mathrm{A}$, et al. Cannabinoids and neuroprotection in basal ganglia disorders. Mol Neurobiol. 2007;36(1):82-91. https://dx.doi. org/10.1007/s12035-007-0004-3.

4. Fernández-Ruiz J. The endocannabinoid system as a target for the treatment of motor dysfunction. Br J Pharmacol. 2009;156(7):1029-40. https://doi.org/10.1111/j.14765381.2008.00088.x.

5. García-Arencibia M, García C, Fernández-RuizJ. Cannabinoids and Parkinson's disease. CNS Neurol Disord Drug Targets. 2009;8(6):432-9. doi: 10.2174/187152709789824642. 
6. Hill AJ, Williams CM, Whalley BJ, Stephens GJ. Phytocannabinoids as novel therapeutic agents in CNS disorders. Pharmacol Ther. 2012;133(1):79-97. doi: 10.1016/j. pharmthera.2011.09.002.

7. Giacoppo S, Mandolino G, Galuppo M, Bramanti P, Mazzon E. Cannabinoids: new promising agents in the treatment of neurological diseases. Molecules. 2014;19(11):18781-816. doi: 10.3390/molecules191118781.

8. Stampanoni Bassi M, Sancesario A, Morace R, Centonze D, Iezzi E. Cannabinoids in Parkinson's Disease. Cannabis Cannabinoid Res. 2017;2(1):21-9. doi: 10.1089/ can.2017.0002.

9. Fernández-Ruiz J, Moreno-Martet M, Rodríguez-Cueto $\mathrm{C}$, Palomo-Garo C, Gómez-Cañas $\mathrm{M}$, et al. Prospects for cannabinoid therapies in basal ganglia disorders. Br J Pharmacol. 2011;163(7):1365-78. https://doi.org/10.1111/ j.1476-5381.2011.01365.x.

10. World Health Organization. Neurological disorders: public health challenges. Genebra: WHO Press; 2006. http://www. who.int/mental_health/publications/neurological_disorders ph_challenges/en/.

11. Sañudo-Peña MC, Patrick SL, Khen S, Patrick RL, Tsou $\mathrm{K}$, et al. Cannabinoid effects in basal ganglia in a rat model of Parkinson's disease. Neurosci Lett. 1998;248(3):171-4. https://doi.org/10.1016/S0304-3940(98)00368-1.

12. Zeng BY, Dass B, Owen A, Rose S, Cannizzaro C, et al. Chronic L-DOPA treatment increases striatal cannabinoid $\mathrm{CB}_{1}$ receptor mRNA expression in 6-hydroxydopamine-lesioned rats. Neurosci Lett. 1999;276(2):71-4. https://doi.org/10.1016/ S0304-3940(99)00762-4.

13. Di Marzo V, Hill MP, Bisogno T, Crossman AR, Brotchie JM. Enhanced levels of endogenous cannabinoids in the globus pallidus are associated with a reduction in movement in an animal model of Parkinson's disease. FASEB J. 2000;14(10):1432-8. https://doi.org/10.1096/ fasebj.14.10.1432.

14. Lastres-Becker I, Cebeira M, de Ceballos ML, Zeng BY, Jenner $\mathrm{P}$, et al. Increased cannabinoid $\mathrm{CB}_{1}$ receptor binding and activation of GTP-binding proteins in the basal ganglia of patients with Parkinson's syndrome and of MPTP-treated marmosets. Eur J Neurosci. 2001;14(11):1827-32. https://doi. org/10.1046/j.0953-816x.2001.01812.x.

15. Silverdale MA, McGuire S, McInnes A, Crossman AR, Brotchie JM. Striatal cannabinoid $\mathrm{CB}_{1}$ receptor mRNA expression is decreased in the reserpine-treated rat model of Parkinson's disease. Exp Neurol. 2001;169(2):400-6. https:// doi.org/10.1006/exnr.2001.7649.

16. Brotchie JM. CB $\mathrm{CB}_{1}$ cannabinoid receptor signalling in Parkinson's disease. Curr Opin Pharmacol. 2003;3(1):54-61. https://doi.org/10.1016/S1471-4892(02)00011-5.

17. González S, Mena MA, Lastres-Becker I, Serrano A, de Yébenes JG, et al. Cannabinoid $\mathrm{CB}(1)$ receptors in the basal ganglia and motor response to activation or blockade of these receptors in parkin-null mice. Brain Res. 2005;1046(1-2):195206. https://doi.org/10.1016/j.brainres.2005.04.010.

18. Farkas S, Nagy K, Jia Z, Harkany T, Palkovits M, et al. The decrease of dopamine $\mathrm{D}_{2} / \mathrm{D}_{3}$ receptor densities in the putamen and nucleus caudatus goes parallel with maintained levels of $\mathrm{CB}_{1}$ cannabinoid receptors in Parkinson's disease: a preliminary autoradiographic study with the selective dopamine $\mathrm{D}_{2} / \mathrm{D}_{3}$ antagonist $\left[{ }^{3} \mathrm{H}\right]$ raclopride and the novel $\mathrm{CB}_{1}$ inverse agonist $\left[{ }^{125} \mathrm{I}\right] \mathrm{SD} 7015$. Brain Res Bull. 2012;87(6):50410. https://doi.org/10.1016/j.brainresbull.2012.02.012.

19. Maneuf YP, Crossman AR, Brotchie JM. The cannabinoid receptor agonist WIN 55,212-2 reduces $\mathrm{D}_{2}$, but not $\mathrm{D}_{1}$, dopamine receptor-mediated alleviation of akinesia in the reserpine-treated rat model of Parkinson's disease. Exp Neurol. 1997;148(1):265-70. https://doi.org/10.1006/ exnr.1997.6645.

20. Fox SH, Henry B, Hill M, Crossman A, Brotchie J. Stimulation of cannabinoid receptors reduces levodopainduced dyskinesia in the MPTP-lesioned nonhuman primate model of Parkinson's disease. Mov Disord. 2002;17(6):11807. https://doi.org/10.1002/mds.10289.

21. Ferrer B, Asbrock N, Kathuria S, Piomelli D, Giuffrida A. Effects of levodopa on endocannabinoid levels in rat basal ganglia: implications for the treatment of levodopa-induced dyskinesias. Eur J Neurosci. 2003;18(6):1607-14. https://doi. org/10.1046/j.1460-9568.2003.02896.x.

22. Cao X, Liang L, Hadcock JR, Iredale PA, Griffith $\mathrm{DA}$, et al. Blockade of cannabinoid type 1 receptors augments the antiparkinsonian action of levodopa without affecting dyskinesias in 1-methyl-4-phenyl-1,2,3,6tetrahydropyridine-treated rhesus monkeys. J Pharmacol Exp Ther. 2007;323(1):318-26. doi: https://doi.org/10.1124/ jpet.107.125666.

23. Morgese MG, Cassano T,Cuomo V, Giuffrida A. Antidyskinetic effects of cannabinoids in a rat model of Parkinson's disease: role of $\mathrm{CB}(1)$ and TRPV1 receptors. Exp Neurol. 2007;208(1):110-9. https://doi.org/10.1016/j. expneurol.2007.07.021.

24. García-Arencibia M, Ferraro L, Tanganelli S, Fernández-Ruiz J. Enhanced striatal glutamate release after the administration of rimonabant to 6-hydroxydopamine-lesioned rats. Neurosci Lett. 2008;438(1):10-3. https://doi.org/10.1016/j. neulet.2008.04.041.

25. van Vliet SA, Vanwersch RA, Jongsma MJ, Olivier B, Philippens IH. Therapeutic effects of Delta9-THC and modafinil in a marmoset Parkinson model. Eur Neuropsychopharmacol. 2008;18(5):383-9. https://doi. org/10.1016/j.euroneuro.2007.11.003.

26. Walsh S, Gorman AM, Finn DP, Dowd E. The effects of cannabinoid drugs on abnormal involuntary movements in dyskinetic and non-dyskinetic 6-hydroxydopamine lesioned rats. Brain Res. 2010;1363:40-8. https://doi.org/10.1016/j. brainres.2010.09.086.

27. Chung YC, Bok E, Huh SH, Park JY, Yoon SH, et al. Cannabinoid receptor type 1 protects nigrostriatal dopaminergic neurons against MPTP neurotoxicity by inhibiting microglial activation. J Immunol. 2011;187(12):6508-17. doi: https://doi. org/10.4049/jimmunol.1102435.

28. García C, Palomo-Garo C, García-Arencibia M, Ramos J, Pertwee R, et al. Symptom-relieving and neuroprotective effects of the phytocannabinoid $\Delta^{9}$-THCV in animal models of Parkinson's disease. Br J Pharmacol. 2011;163(7):1495-506. https://doi.org/10.1111/j.1476-5381.2011.01278.x.

29. Martinez A, Macheda T, Morgese MG, Trabace L, Giuffrida 
A. The cannabinoid agonist WIN55212-2 decreases L-DOPAinduced PKA activation and dyskinetic behavior in 6-OHDAtreated rats. Neurosci Res. 2012;72(3):236-42. https://doi. org/10.1016/j.neures.2011.12.006.

30. Gutiérrez-Valdez AL, García-Ruiz R, Anaya-Martínez V, Torres-Esquivel C, Espinosa-Villanueva J, et al. The combination of oral L-DOPA/rimonabant for effective dyskinesia treatment and cytological preservation in a rat model of Parkinson's disease and L-DOPA-induced dyskinesia. Behav Pharmacol. 2013;24(8):640-52. DOI: 10.1097/FBP.0000000000000004.

31. Price DA, Martinez AA, Seillier A, Koek W; Acosta Y, et al. WIN55,212-2, a cannabinoid receptor agonist, protects against nigrostriatal cell loss in the 1-methyl-4-phenyl1,2,3,6-tetrahydropyridine mouse model of Parkinson's disease. Eur J Neurosci. 2009;29(11):2177-86. https://doi. org/10.1111/j.1460-9568.2009.06764.x.

32. Carroll CB, Zeissler ML, Hanemann CO, Zajicek JP. $\Delta^{9}$ tetrahydrocannabinol $\left(\Delta^{9}\right.$-THC) exerts a direct neuroprotective effect in a human cell culture model of Parkinson's disease. Neuropathol Appl Neurobiol. 2012;38(6):535-47. https://doi. org/10.1111/j.1365-2990.2011.01248.x.

33. Sieradzan KA, Fox SH, Hill M, Dick JP, Crossman $A R$, et al. Cannabinoids reduce levodopa-induced dyskinesia in Parkinson's disease: a pilot study. Neurology. 2001;57(11):2108-11. doi: https://doi.org/10.1212/ WNL.57.11.2108.

34. García-Arencibia M, González S, de Lago E, Ramos JÁ, Mechoulam R, et al. Evaluation of the neuroprotective effect of cannabinoids in a rat model of Parkinson's disease: importance of antioxidant and cannabinoid receptorindependent properties. Brain Res. 2007;1134(1):162-70. https://doi.org/10.1016/j.brainres.2006.11.063.

35. Little JP, Villanueva EB, Klegeris A. Therapeutic potential of cannabinoids in the treatment of neuroinflammation associated with Parkinson's disease. Mini Rev Med Chem. 2011;11(7):582-90. doi: 10.2174/138955711795906905.

36. Gómez-Gálvez Y, Palomo-Garo C, Fernández-Ruiz J, García C. Potential of the cannabinoid $\mathrm{CB}(2)$ receptor as a pharmacological target against inflammation in Parkinson's disease. Prog Neuropsychopharmacol Biol Psychiatry. 2016;64:200-8. https://doi.org/10.1016/j.pnpbp.2015.03.017.

37. Zuardi AW. Cannabidiol: from an inactive cannabinoid to a drug with wide spectrum of action. Rev Bras Psiquiatr. 2008;30(3):271-80. http://dx.doi.org/10.1590/S151644462008000300015 .

38. Zhornitsky S, Potvin S. Cannabidiol in humans-the quest for therapeutic targets. Pharmaceuticals. 2012;5(5):529-52. https://doi.org/10.3390/ph5050529.

39. Iuvone T, Esposito G, De Filippis D, Scuderi C, Steardo L. Cannabidiol: a promising drug for neurodegenerative disorders? CNS Neurosci Ther. 2009;15(1):65-75. https:// doi.org/10.1111/j.1755-5949.2008.00065.x.

40. Leweke FM, Piomelli D, Pahlisch F, Muhl D, Gerth CW, et al. Cannabidiol enhances anandamide signaling and alleviates psychotic symptoms of schizophrenia. Transl Psychiatry. 2012;2:e94. http://dx.doi.org/10.1038/tp.2012.15.

41. Zuardi AW, Crippa JA, Hallak JE, Bhattacharyya S, Atakan Z, et al. A critical review of the antipsychotic effects of cannabidiol: 30 years of a translational investigation. Curr Pharm Des. 2012;18(32):5131-40. doi: 10.2174/138161212802884681.

42. Schubart CD, Sommer IE, Fusar-Poli P, de Witte L, Kahn
RS, et al. Cannabidiol as a potential treatment for psychosis. Eur Neuropsychopharmacol. 2014;24(1):51-64. https://doi. org/10.1016/j.euroneuro.2013.11.002.

43. Lastres-Becker I, Molina-Holgado F, Ramos JA, Mechoulam R, Fernández-Ruiz J. Cannabinoids provide neuroprotection against 6-hydroxydopamine toxicity in vivo and in vitro: relevance to Parkinson's disease. Neurobiol Dis. 2005;19(12):96-107. https://doi.org/10.1016/j.nbd.2004.11.009.

44. Dos-Santos-Pereira M, da-Silva CA, Guimarães FS, Del-Bel E. Co-administration of cannabidiol and capsazepine reduces L-DOPA-induced dyskinesia in mice: Possible mechanism of action. Neurobiol Dis. 2016;94:179-95. https://doi. org/10.1016/j.nbd.2016.06.013.

45. Fox SH, Kellett M, Moore AP, Crossman AR, Brotchie JM. Randomised, double-blind, placebo-controlled trial to assess the potential of cannabinoid receptor stimulation in the treatment of dystonia. Mov Disord. 2002;17(1):145-9. https://doi.org/10.1002/mds.1280.

46. Carroll CB, Bain PG, Teare L, Liu X, Joint C, et al. Cannabis for dyskinesia in Parkinson disease: a randomized doubleblind crossover study. Neurology. 2004;63(7):1245-50. doi: https://doi.org/10.1212/01.WNL.0000140288.48796.8E.

47. Mesnage V; Houeto JL; Bonnet AM; Clavier I; Arnulf I et al. Neurokinin B, neurotensin, and cannabinoid receptor antagonists and Parkinson disease. Clin Neuropharmacol. 2004;27(3):108-10.

48. Venderová K, Růzicka E, Vorísek V, Visnovský P. Survey on cannabis use in Parkinson's disease: subjective improvement of motor symptoms. Mov Disord. 2004;19(9):1102-6. https:// doi.org/10.1002/mds.20111.

49. Finseth TA, Hedeman JL, Brown RP 2nd, Johnson KI, Binder MS, et al. Self-reported efficacy of cannabis and other complementary medicine modalities by Parkinson's disease patients in Colorado. Evid Based Complement Alternat Med. 2015;2015:874849. https://dx.doi.org/10.1155/2015/874849.

50. Balash Y, Bar-Lev Schleider L, Korczyn AD, Shabtai H, Knaani J, et al. Medical cannabis in Parkinson disease: real-life patients' experience. Clin Neuropharmacol. 2017;40(6):268272. http://insights.ovid.com/pubmed?pmid=29059132.

51. Lotan I, Treves TA, Roditi Y, Djaldetti R. Cannabis (medical marijuana) treatment for motor and non-motor symptoms of Parkinson disease: an open-label observational study. Clin Neuropharmacol. 2014;37(2):41-4. doi: 10.1097/ WNF.0000000000000016.

52. Zuardi AW, Crippa JA, Hallak JE, Pinto JP, Chagas MH, et al. Cannabidiol for the treatment of psychosis in Parkinson's disease. J Psychopharmacol. 2009;23(8):979-83. https://doi. org/10.1177/0269881108096519.

53. Chagas MH, Eckeli AL, Zuardi AW, Pena-Pereira MA, Sobreira-Neto MA, et al. Cannabidiol can improve complex sleep-related behaviours associated with rapid eye movement sleep behaviour disorder in Parkinson's disease patients: a case series. J Clin Pharm Ther. 2014;39(5):564-6. https://doi. org/10.1111/jcpt.12179.

54. Chagas MH, Zuardi AW, Tumas V, Pena-Pereira MA, Sobreira ET, et al. Effects of cannabidiol in the treatment of patients with Parkinson's disease: an exploratory double-blind trial. J Psychopharmacol. 2014;28(11):1088-98. https://doi. org/10.1177/0269881114550355.

Recebido:

Aceito: 